\title{
Understanding How Microbiomes Influence The Systems They Inhabit: Moving From A Correlative To A Causal Research Framework
}

Authors: Hall, E.K. ${ }^{*}$, Bernhardt, E.S ${ }^{2}$, Bier, R.L. ${ }^{2}$, Bradford, M.A. ${ }^{3}$, Boot, C.M. ${ }^{1}$, Cotner, J.B. ${ }^{4}$, del Giorgio, P.A. ${ }^{5}$, Evans, S.E. ${ }^{6}$, Graham E. B ${ }^{2,7.8}$, Jones, S.E. ${ }^{9}$, Lennon, J.T. ${ }^{10}$, Locey, K.J. ${ }^{10}$ Nemergut, D. ${ }^{2}$, Osborne, B.B. ${ }^{11}$, Rocca, J.D. ${ }^{1,2}$, Schimel J.S. $^{12}$, Waldrop, M.P. ${ }^{13}$, Wallenstein, M.W. ${ }^{1}$

Article Type: Perspectives

Words in Abstract: 262

Words in Main Text: 4,238

2 Figures, 0 Tables, 45 References

Running Title: How microbiomes influence ecosystems

Keywords: microbiome, microbial ecology, ecosystem ecology, emergent properties, community aggregated traits

Affiliations: ${ }^{1}$ Colorado State University, Fort Collins CO (ed.hall@colostate.edu, claudia.boot@colostate.edu, matt.wallenstein@colostate.edu) 2Duke University, Durham, N.C. (ebernhar@duke.edu, ebgraham2@colorado.edu, jennifer.rocca@duke.edu) ${ }^{3}$ Yale University, New Haven, CT, Dept. of Forestry and Environmental Studies (mark.bradford@yale.edu), ${ }^{4}$ University of Minnesota, Saint Paul, MN (cotne002@umn.edu) ${ }^{5}$ Université du Québec à Montréal, Montréal, CA (del_giorgio.paul@uqam.ca) ${ }^{6}$ Michigan State University, Hickory Corners, MI (evanssar@gmail.com) ${ }^{7}$ Institute of Arctic and Alpine Research, University of Colorado at Boulder ${ }^{8}$ Pacific Northwest National Laboratory, Richland, WA, USA ${ }^{9}$ University of Notre Dame, South Bend, IN (sjones20@nd.edu) ${ }^{10}$ Indiana University, Bloomington, IN (lennonj@indiana.edu, ken@weecology.org) ${ }^{11}$ Brown University, Providence, RI (brooke_osborne@brown.edu) ${ }^{12}$ University of California, Santa Barbara, Santa Barbara, CA (josh.schimel@lifesci.ucsb.edu) ${ }^{13}$ U.S. Geological Survey, Menlo Park, CA (mwaldrop@usgs.gov)

* Corresponding Author: Dr. Ed Hall, Department of Ecosystem Science and Sustainability, Natural Resource Ecology Laboratory Campus Delivery 1499, Colorado State University, Fort Collins, CO 80523, ed.hall@colostate.edu, 970-4912162

Contribution: All listed authors have contributed to the conceptualization, writing, and preparation of the current manuscript. 


\section{Abstract}

2 Translating the ever-increasing wealth of information on microbiomes (environment,

3 host, or built environment) to advance the understanding of system-level processes

4 is proving to be an exceptional research challenge. One reason for this challenge is

5 that relationships between characteristics of microbiomes and the system-level

6 processes they influence are often evaluated in the absence of a robust conceptual

7 framework and reported without elucidating the underlying causal mechanisms. The

8 reliance on correlative approaches limits the potential to expand the inference of a

9 single relationship to additional systems and advance the field. We propose that

10 research focused on how microbiomes influence the systems they inhabit should

11 work within a common framework and target known microbial processes that

12 contribute to the system-level processes of interest. Here we identify three distinct

13 categories of microbiome characteristics (microbial processes, microbial community

14 properties, and microbial membership) and propose a framework to empirically link

15 each of these categories to each other and the broader system level processes they

16 affect. We posit that it is particularly important to distinguish microbial community

17 properties that can be predicted from constituent taxa (community aggregated traits)

18 from and those properties that are currently unable to be predicted from constituent

19 taxa (emergent properties). Existing methods in microbial ecology can be applied to

20 more explicitly elucidate properties within each of these categories and connect

21 these three categories of microbial characteristics with each other. We view this

22 proposed framework, gleaned from a breadth of research on environmental

23 microbiomes and ecosystem processes, as a promising pathway with the potential to

24 advance discovery and understanding across a broad range of microbiome science. 


\section{Current Approaches Linking Microbial Characteristics and Ecosystem}

\section{Processes}

27 Virtually all ecosystem processes are influenced by microorganisms, and many processes are carried out exclusively by microorganisms. This has sometimes led to the assumption that a better description of the microbiome (including its associated transcripts, proteins, and metabolic products) should lead to a better understanding and predictions of system level processes. However, such justifications assume that measurable characteristics of the microbiome (e.g. 16S rRNA gene libraries, metagenomes, enzymatic activities) can inform our ability to better understand and predict system-level processes. Unfortunately, additional information about the microbiome does not always provide a clearer understanding of ecosystem processes beyond what can be predicted by environmental factors alone ${ }^{1,2}$. Two recent meta-analyses ${ }^{3,4}$ suggest that research at the intersection of ecosystem science and microbial ecology often relies on assumed relationships between microbiome characteristics and ecosystem processes and often do not test to see if those relationships are present. The first, an examination of 415 studies, found little evidence that protein-encoding genes (sometimes referred to as "functional genes") or gene transcripts correlate with associated biogeochemical processes $^{3}$. Although all studies attempted (or presumed) to link microbial genes or

44 transcripts with function, only $14 \%$ measured both the abundance of genes or transcripts and the corresponding process. When the relationship between microbial characteristic and ecosystem process was measured and tested only $38 \%$ exhibited a positive correlation ${ }^{3}$. This result was consistent whether functional gene or transcript abundance was used as the response variable. 
49 The second study compiled a separate dataset of 148 studies that examined microbial membership and ecosystem processes in response to experimental manipulations ${ }^{4}$. Whereas $40 \%$ of included studies reported concomitant changes in microbial membership and an ecosystem process, only one third of those cases reported the relationship between microbial membership and an ecosystem process. Interestingly, of the 53 studies that posed a hypothesis about links between microbial membership and ecosystem processes, more than half $(53 \%)$ did not report testing for a statistical link between membership and processes $^{4}$

Microbiomes are the engines that power system-level processes ${ }^{5}$. However the meta-analyses described above illustrate that the current approach to study the links between microbiome characteristics and ecosystem processes are not well formulated and relationships between microbiome characteristics and system level processes are implied yet rarely tested. When linkages are tested, significant correlations between microbiome characteristics and ecosystem processes are sometimes present, but more frequently not ${ }^{3,4}$. One reason for the ambiguity between microbiome characteristics and system level processes is that many studies are conducted in the absence of a conceptual framework that illustrates how different measurable microbial characteristics relate to one another and to the system level process of interest.

\section{Challenges in Linking Microbial Characteristics and Ecosystem Processes} Current conceptual research frameworks that attempt to link microbial information to a system-level process often do not effectively align with the methods being applied or the data those methods generate. For example, environmental factors act on the physiology of individual organisms, which alters their competitive ability, 
abundance, and ultimately their contribution to an ecosystem process. However, designing an observational study or experiment from this framework assumes that environmental and microbial characteristics are measurable across multiple categories of ecological organization (i.e., individuals, populations, and communities) at the temporal and spatial scales at which they influence system level processes (Figure 1a). In addition, the relationships between environmental variables and microbial characteristics can be decoupled in both time and space ${ }^{4}$, and are often non-linear ${ }^{6}$. Recent immigration, phenotypic plasticity, disequilibrium between the environment and the extant microbiome at the time of sampling, functional redundancy, and dormancy can all mask the relationship between measurable microbial characteristics and the processes microorganisms influence (Figure 1b). ${ }^{4,7,8,9}$ As micrometer scale characteristics of microbiomes $\left(10^{-6} \mathrm{~m}\right)$ are scaled to the level of ecosystems ( $\mathrm{m}$ to $\mathrm{km})$, we assume that our conceptual understanding is also scalable. However, each of the aforementioned confounding factors aggregate over multiple orders of magnitude often masking the very relationships we seek to elucidate (Figure 1b). To formalize how measurable microbiome characteristics are linked with system-level processes we have conceptually defined the intersection of microbial and ecosystem ecology and identified three categories of microbial characteristics to illustrate how they interact with each other and may contribute to an ecosystem process (Figure 2).

\section{Mapping Ecosystem Processes to Microbial Characteristics}

Defining the ecosystem process, its critical sub-processes, and the known phylogenetic distribution of the metabolic pathways that drive those sub-processes creates an explicit conceptual pathway that links the ecosystem process to the microorganisms that contribute to it. Ecosystem processes are defined as qualitative 
changes in a pool, or a flux from one pool to another (e.g., $\mathrm{NH}_{4}{ }^{+}$to $\mathrm{NO}_{3}{ }^{-}$, or dissolved organic matter to $\mathrm{CO}_{2}$ ). The first step to understand how a microbiome influences an ecosystem process is to define the ecosystem process of interest and each subprocess that contributes to it (i.e., the set of constituent reactions that combine to determine a net flux). Ecosystem processes are composites of complementary or antagonistic sub-processes, carried out by phylogenetically and metabolically diverse microorganisms ${ }^{10}$. For example, net ecosystem productivity (NEP) is the balance between antagonistic processes of C-fixation and C-mineralization. Each sub-process of NEP can be further partitioned into a series of metabolic pathways (e.g., chemoautotrophic nitrification and photoautotrophic C-fixation or heterotrophic

109 fermentation and aerobic respiration). Partitioning each ecosystem process in this

110 hierarchical manner can continue until the sub-processes maps directly to specific

111 microbial metabolic pathways (e.g., acetoclastic methanogenesis). Subsequently,

112 each of these metabolic pathways can be categorized as either phylogenetically

113 broad or narrow ${ }^{11}$. Broad processes are phylogenetically common (i.e., widely

114 distributed among taxa), whereas narrow processes are phylogenetically conserved

115 (i.e., limited to a specific subset of taxa). For example, denitrification and

116 photosynthesis are phylogenetically broad processes, while both methanogenesis

117 and methanotrophy are currently thought to be phylogenetically narrow processes

118 (with at least one notable exception ${ }^{12}$ ).

119 The second step is to identify the controls or constraints on each constituent subprocess. For example, the kinetics of a single metabolic pathway in a model

121 organism may help us understand the rate limiting steps of a narrow process, but

122 insights from model organisms are much less likely to capture the full spectrum of

123 responses of a broad process where phenotypic variation among phylogenetically 
124 diverse organisms is likely to be much greater ${ }^{13,14}$. Once the ecosystem process has

125 been conceptually partitioned into its component parts and their primary controls, a

126 concerted approach can be applied to investigate how characteristics of the

127 microbiome influence the ecosystem process of interest within the complexity of a

128 natural environment.

\section{Categories of Microbial Characteristics}

130 The contribution of the microbiome to ecosystem processes is exerted through

131 aggregate community properties that are shaped by both microbial membership and

132 environmental factors. Most current studies rarely articulate how measurements of

133 microbial characteristics differ in their specificity (i.e., the level of phylogenetic

134 resolution), precision (i.e., the ability of the method to repeatedly describe the

135 characteristic of interest), or context (i.e., how a characteristic relates to other

136 microbial characteristics or the ecosystem). To aid in designing experiments that link

137 microorganisms to the processes they influence we propose three distinct categories

138 of microbial characteristics: 1) microbial processes, 2) microbial community

139 properties, and 3) microbial membership (Figure 2). This proposed framework allows

140 the researcher to clearly identify how different measurements used to characterize a

141 microbiome interact with each other, and to identify the potential of each

142 characteristic to elucidate the microbial contribution to the system level process. All

143 measurable characteristics of microbial communities (e.g., abundance of cells,

144 sequence of genes, transcripts, or proteins; enzyme expression or activity) can be

145 placed within one of the above categories. This conceptual structure that orientates

146 each microbial category within a broader context creates the opportunity to improve

147 the design of observational and experimental studies in microbiome research. 
Microbial Processes - Microbial processes are the collective metabolisms of the

149 microbiome that contribute to changes in pools and fluxes of elements or compounds

150 (i.e., Figure 2, Letter K). This is the category of microbial information that can most

151 readily be incorporated into system-level models because many microbial processes

152 represent the key sub-processes that contribute to a particular ecosystem process

(e.g., methanogenesis + methanotrophy $\approx$ methane efflux). Commonly measured

microbial processes in ecosystem science include nitrogen fixation, denitrification,

nitrification, phosphorus uptake and immobilization, carbon fixation, and organic

carbon mineralization. The rates of many microbial processes can be approximated

157 through physiological assays (e.g., biological oxygen demand to estimate microbial respiration), and while they do not open the "black box" of the microbial community, they do directly quantify the microbial contribution (or potential contribution) to changes in resources moving through the box. Microbial processes can be distinguished from other microbial characteristics because they are all rates (i.e., have time in the denominator) and require a bioassay to estimate.

163 Assays used to estimate microbial processes are often logistically challenging, require manipulations that inevitably deviate from in situ conditions, and often depend on the environment from which the microbiome was sampled. For example,

166 the relationship between temperature and microbial processes such as enzyme

167 activity and phosphorus use efficiency vary across latitudinal gradients ${ }^{15}$ and among 168 seasons $^{16}$, respectively. Thus, observations of the effect of temperature on either enzyme activity or phosphorus use efficiency depend on where (e.g., at what latitude) and when (e.g., during which season) they were measured. In the absence

171 of an understanding of the underlying physiological mechanism (e.g., the

172 physiological change that allows a community to perform differently at different 
temperatures), the relationship between and environmental driver (e.g., temperature) and a microbial process must be measured through a direct assay at each location and at each time. This limits the inference possible from relying on measurements of

176 microbial processes alone to understand the microbial contribution to an ecosystem

177 process.

178 Microbial Community Properties - Microbial community properties include a broad set of microbial characteristics such as community biomass or biomass elemental ratios (e.g., biomass $\mathrm{C}: \mathrm{N}$ or $\mathrm{C}: \mathrm{P}$ ratios) and the majority of phylogenetically undifferentiated aggregate sequence based measurements (e.g., gene abundance, metagenomes, transcriptomes). Microbial community properties (Figure 2) represent an integrated characteristic of the microbiome that has the potential to predict or at least constrain the estimates of microbial processes. For example, microbial community biomass C:N (a community property) has been shown to indicate a microbiome's potential to mineralize or immobilize $\mathrm{N}$ in terrestrial ${ }^{17}$, freshwater ${ }^{18}$ and 187 marine ${ }^{19}$ ecosystems.

188 Microbial community properties can be separated into two categories, emergent properties and community aggregated traits. It is generally agreed that emergent properties refer to a quality of the whole that is unique and distinguishable from the additive properties of its constituents. Whereas in some cases, emergent properties may be predicted from their constituent parts, for example, prediction of the physical

193 properties of carbon polymers is possible based on the atomic organization of 194 carbon atoms within each structure ${ }^{20}$, in microbial ecology our understanding of the constituent parts and their interactions are more often than not insufficient to predict emergent properties. Here we use emergent properties as it has been defined

197 previously in ecology ${ }^{21}$ : "An emergent property of an ecological unit is one which is 
wholly unpredictable from observation of the components of that unit", which is also consistent with its contemporary use in microbial ecology 22 .

200 Emergent properties of microbiomes influence important ecosystem processes. For example, a series of experiments in flow-through flumes mimicked development and metabolism of stream biofilms ${ }^{23}$. Transient storage (i.e., an increase in residence time of the water and its solutes near the biofilm relative to the flow around it) increased as the microbial biofilm density increased..$^{23}$ In this case biofilm density was an emergent property of the microbiome and transient storage was the microbial process it mediated. Another example of an emergent property is the distribution of traits that influences key microbial processes. Trait based approaches have a rich history in ecology and have been increasingly applied to address questions in multiple areas of microbial ecology. ${ }^{24}$ For example, uptake of an organic substrate can often involve the expression of multiple genes, differing among individual

211 organisms, all capable of performing uptake of the organic substrate, albeit with

212 differences in the underlying efficiency. The distribution of the expression of these

213 functional gene variants generates a trait distribution that is an emergent property of

214 the microbiome. That emergent trait distribution determines the overall performance

215 of the microbiome for that function (i.e., the uptake of the organic substrate), but it

216 cannot be predicted from the presence of the organisms conferring that trait using

217 current methods. ${ }^{25}$ While characterization of emergent properties may improve the

218 understanding of microbial processes (Figure 2, Letter G) currently they most often

219 cannot be estimated or predicted on the basis of the constituent taxa (i.e.

220 membership) alone (Figure 2, Letter F), and thus must remain as an intermediary

221 between environmental drivers (Figure 2, Letter $\mathrm{C}$ ) and microbial processes (Figure

2, Letter G). 
Unlike emergent properties, community aggregated traits can potentially be estimated from characteristics of their constituents and provide a pathway to link microbial community membership to the community properties that drive microbial processes (Figure 2, Letter E). ${ }^{26}$ For example, community aggregated traits may include commonly measured community properties such as functional gene abundance as estimated from qPCR (e.g., pmoA which encodes a subunit of the enzyme involved in methane oxidation, can be used to estimate potential for methanotrophy and as a phylogenetic marker for methanotrophs) ${ }^{27}$. A recent perspective article that discussed the role of community aggregated traits in microbial ecology noted a series of additional putative community aggregated traits

233 (e.g., maximum growth rate, dormancy, osmoregulation) that could be inferred from

234 metagenomic data of the extant community. ${ }^{26}$

235 Understanding which community properties can be predicted by membership is a 236 critical research question and an important step in understanding how the

237 microbiome contributes to system level processes. Whether or not a community

238 property is likely to be an emergent property or a community aggregated trait is an exciting area of research and provides an important framework to advance research at the microbial-ecosystem nexus. New approaches, like studying higher-level interactions in ecological communities could help understand how a microbiome's

242 constituents interact to from emergent properties. ${ }^{28}$ This is not a trivial task, yet a 243 suite of existing methods, discussed below, provides the ability to directly pursue this 244 challenge. Microbial Community Membership - Although the now commonplace analysis of 246 community membership by sequencing phylogenetic markers (e.g., ITS regions or 247 regions of the16S rRNA and 18S rRNA genes) or suites of phylogenetically 
conserved protein sequences identifies constituent microbial taxa, the direct coupling

249 of microbial phylogeny to physiology and ecology remains elusive (Figure 2, Letter

H). ${ }^{29,30,31}$ In general the paucity of associated physiological data or information on

251 population phenotypes that accompany phylogenetic analyses limits the system-level

252 inference that is possible from analyses of community membership. Even when the physiology of an organism is known, it appears many metabolic pathways are phylogenetically broad, and that any given microbiome will contain a diverse set of microorganisms with the genes that encode many of the same common microbial metabolic pathways, often referred to as "functional redundancy" 32 . There also appears to be no consistent phylogenetic resolution at which specific microbial metabolic pathways are constrained ${ }^{32}$. This constrains our ability to attribute microbial processes to community membership of even relatively simple environmental consortia. Whereas it is clear that microbial populations are not

261 randomly distributed in space and time ${ }^{31}$, and that some microbial traits are

262 conserved at coarse taxonomic scales, ${ }^{24,33,34}$ the physiological mechanisms

263 underlying non-random distributions of microbial taxa across environmental

264 gradients are often unknown. This limited understanding of the metabolism of most bacterial phyla is one thing that currently prevents linking a microorganism's

266 abundance in an environment to its role in a related microbial process.

\section{A Path Forward}

268 We suggest that a challenging but necessary step for microbiome science is to move away from identifying correlative relationships between characteristics of the microbiome and system level processes, and towards identifying more causative and

271 mechanistic relationships. The conceptual diagram (Figure 2) is a road map to

272 organize and link the diverse suite of measurable microbial characteristics that are 
currently available to researchers. Figure 2 does not represent how these components necessarily interact in the environment; rather it is a map that identifies potential links between measurable microbial and system-level characteristics that can help structure our exploration of how microorganisms influence the systems they inhabit. Ecosystem ecology has traditionally been confined to interactions between environmental parameters and ecosystem processes (depicted within the horizontal arrow, Figure 2). Similarly, microbial ecology (depicted within the vertical arrow, communities (e.g., bacterial abundance) and microbial processes (e.g., bacterial production), or the physiology of microbial isolates (e.g., sulfate reducing bacteria) or the collective physiology of highly reduced communities with known membership approaches in studies of environmental microorganisms has led to an increasingly detailed description of the world's microbiomes and an increasing interest in how constituents of those microbiomes interact to influence the system as a whole. Currently, researchers use a range of approaches for attempting to link characteristics of the microbiome to ecosystem processes. Direct connections between microbial membership and ecosystem processes (Figure 2, Letter I), or community properties and ecosystem processes (Figure 2, Letter J), have proven

292 difficult to establish. ${ }^{3,4}$ We propose 1) identifying which microbial processes are likely

293 to contribute to ecosystem-level pools and fluxes a priori (Figure 2, Letter K), 2)

294 determining which microbial community properties best describe and predict these

295 microbial processes (Figure 2, Letter G), and 3) identifying whether the community

296 properties that best describe each process are a community aggregated trait or a

297 emergent property (Community Properties, Figure 2). If the community property is 
298 likely to be a community aggregated trait then exploring the link between microbial

299 membership and community properties may lead to further understanding and

300 perhaps an enhanced predictive power (Figure 2, Letter E). However, if the

301 community property is likely an emergent property elucidating the microbial

302 membership that contributes to the emergent property is, given the current understanding, unlikely to improve understanding of the drivers of that community

304 property (Figure 2, Letter F). Formalizing microbiome research into a structured, links between microbiome characteristics and system-level processes that are most

307 likely to be detected empirically. This approach will also allow researchers working in different systems to test the same pathways among defined microbiome characteristics and thus increase the possibility of understanding the causal mechanism (or absence of causality) for observed correlations. Thus, future

311 research endeavors will be most powerful if they focus on elucidating connections

312 through the complete path of microbial ecology (Figure 2, blue arrow, Letters E, F,

313 and $\mathrm{G}$ ) and not direct connections between microbial membership or community

314 properties and ecosystem processes (Figure 2, Letters I and J).

\section{Applying and Testing the Proposed Framework}

316 Applying and testing the proposed framework will depend on the ability to more

317 robustly characterize each category of microbial characteristics and to directly

318 measure the arrows that connect each category (Figure 2). Both labeling/sorting

319 approaches and phenotypic description of isolates provide an opportunity to better

320 understand how microbial membership contributes to community properties (Figure

321 2, Letter E or F). Labeling and cell sorting approaches (e.g., fluorescent in situ

322 hybridization (FISH) coupled with flow cytometry cell sorting, ${ }^{35}$ or immunocapture, 
323 such as with bromodeoxyuridine, $\mathrm{BrdU})^{36}$ provide powerful tools to constrain the complexity of the microbiome and directly test hypotheses that link membership to community properties or microbial processes. For example, a study of an Arctic

326 Ocean bacterial community labeled the actively growing component of the community using BrdU and then separated those populations from the rest of the community using an immunocapture technique to better understand the portion of the microbiome that was contributing to secondary production. ${ }^{36}$ In addition, physiological studies of isolates that are representative of important community properties have the potential to advance understanding of the role of phenotypic plasticity in structuring how constituent populations do or do not contribute to community properties (Figure 2, Letter E) ${ }^{13}$. Detailed studies of isolates of common environmental OTUs have demonstrated immense variation within a given OTU (i.e., "microdiversity") that in part explains the challenge of linking membership to a community property ${ }^{14}$. For example, work on Prochlorococcus has led to a better understanding of how ecotypes within a single taxonomic unit (OTU) can lead to specialization in temperature, and substrate affinity ${ }^{37}$. OTUs that form a substantial portion of the microbiome's sequence abundance provide potential candidates for

340 further investigation of possible phenotypic plasticity and or microdiversity ${ }^{14}$. For example, a single phylotype of the class Spartobacteria within the phyla

342 Verrucomicrobia was found to be present in a broad range of soil ecosystems and 343 comprised as much as $31 \%$ of all $16 \mathrm{~S}$ rRNA gene sequences returned from prairie soils $^{38}$, making it an excellent candidate for targeted isolation and physiological studies. Studies of environmental isolates are essential in building a broader

346 understanding of how community membership does or does not contribute to

347 community properties (Figure 2, Letters E and F). 
In addition to a better description of each category of microbial characteristics, an

349 important step in moving from a correlative and descriptive approach to a causative and mechanistic approach comes in measuring the arrows represented by letters in

Figure 2. There is a suite of powerful methods already being employed in microbial ecology that can actively measure many of the arrows illustrated in Figure 2, including: stable isotope probing of mixed communities ${ }^{39}$, single cell methods that can assay cells in the physiological state they occur in in the environment, and labeling individual cells with stable isotopes for single cell analyses ${ }^{40}$. Studies that use stable isotope probing or any form of tracking isotopically labeled substrates into a population have been successful in linking microbial membership to microbial processes (Figure 2, Letter H). For example, a study of a Scottish peatland used SIP to reveal that a single species of Desulfosporosinus was most likely responsible for the totality of sulfate reduction within the peatland even though it only comprised $0.006 \%$ of the retrieved $16 S$ rRNA gene sequences ${ }^{41}$. In addition to this example of using SIP to link microbial membership and microbial processes (Figure 2, Letter H), there is a suite of cultivation independent techniques (such as Raman microspectroscopy (MS), NanoSIMS, or energy dispersive spectroscopy, EDS) that complement sequence-based microbiome analyses by reporting on the physiological and phenotypic characteristics of individual cells in situ. ${ }^{40,42,43}$ Both Raman MS and

367 NanoSims can be coupled with a range of in situ hybridization techniques (e.g.,

368 fluorescent in situ hybridization, $\mathrm{FISH}$ ) to identify which populations are contributing 369 to community properties (Figure 2, Letter E) or microbial processes (Figure 2, Letter H). For example, a study of a microbial consortia from the Sippewissett Salt Marsh

371 on the coast of Massachusetts, USA used a combination of FISH and NanoSIMs to 372 confirm a syntrophic association between a population of autotrophic purple sulfur 
373 bacteria and heterotrophic sulfate reducing bacteria $(\mathrm{SRB})^{44}$. These existing

374 methods of confirmatory ecophysiology allowed for direct measurements of the arrows connecting membership with microbial processes (in this case both carbon

376 fixation and sulfate reduction, Figure 2, Letter $\mathrm{H}$ ) in a stable microbial consortium.

377 These cultivation independent approaches also create the potential to begin to determine which community properties are emergent properties, and which are community aggregated traits. For example, microbial community biomass stoichiometry (e.g., biomass C:N or C:P) cannot currently be predicted (or even constrained) from a list of its constituent taxa (Figure 2, Letter F). However, microbial biomass stoichiometry is a community property with power to predict the microbial contribution to nutrient cycling (Figure 2, Letter G). ${ }^{17-19}$ Energy dispersive spectroscopy (EDS) has the power to measure the $\mathrm{C}: \mathrm{N}: \mathrm{P}$ of individual bacterial cells growing in situ (i.e. not in culture) ${ }^{43}$. The potential to couple EDS analysis with a phylogenetic label presents the opportunity to assay mixed microbial communities and assess the link between phylogenetic identity and biomass stoichiometry under natural conditions ${ }^{45}$. This approach would provide a direct link between community membership and a community property (e.g., biomass C:N, Figure 2, Letter E), that influences an important microbial process (i.e., nutrient recycling). These approaches applied in concert with sequence-based analyses have the potential to empirically link the categories of microbial information defined here (Figure 2) with

393 the processes they influence, moving microbiome science from a descriptive and correlative approach to a mechanistic and causative approach.

\section{Designing microbiome research}

396 The framework presented here provides one approach to formalize inquiry across

397 microbiome science and encourages empirical linkages between the presence of 
organisms in a system and the processes that characterize that system. Whereas we draw examples from environmental microbiomes and the ecosystems they inhabit,

400 this structured approach has the potential to benefit the analysis of microbiomes

401 associated with other systems such as host organisms and those of the built

402 environment. As important as establishing causal links among microbial

403 membership, community properties, microbial processes, and ecosystem processes,

404 is determining when these links are unlikely to be present. Research that

405 indiscriminately seeks to identify correlations, which places all microbial

406 characteristics on an equal plane and does not explicitly recognize the relationships

407 between microbial characteristics, are likely to continue to yield conflicting and

408 ambiguous results that not only fail to provide new insight into ecosystem processes,

409 but also blur the connections that do exist. We suggest that rather than looking for

410 linkages among microbiome membership and system-level processes in every study,

411 research efforts would benefit from strategically targeting the linkages and processes

412 for which an a priori understanding of microbial physiology should allow us to

413 improve our understanding of the ecosystem process. 


\section{Acknowledgements}

415 This work is a product of the Next Generation of Ecosystem Indicators Working

416 Group, supported by the USGS John Wesley Powell Center for Synthesis and

417 Analysis. Preparation of this manuscript was supported by NSF DEB IOS \#1456959

418 awarded to EKH. Chuck Pepe-Ranney and Ariane Peralta provided valuable

419 feedback on previous versions of this manuscript. This paper is dedicated to Diana

420 Nemergut, an integral part of our working group who passed away during the

421 preparation of this manuscript. She is one of a kind. 


\section{Figures}

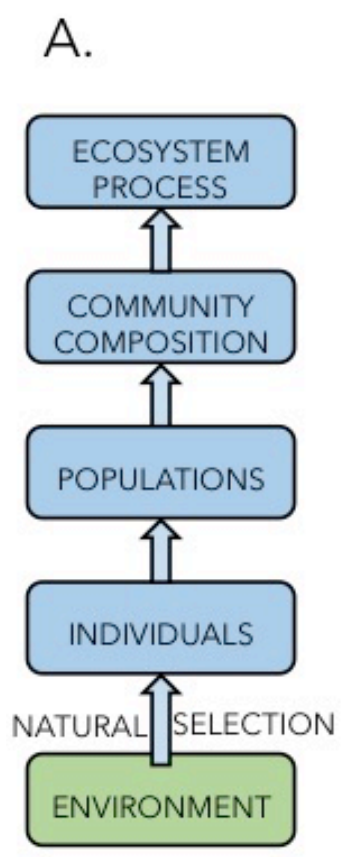

B.

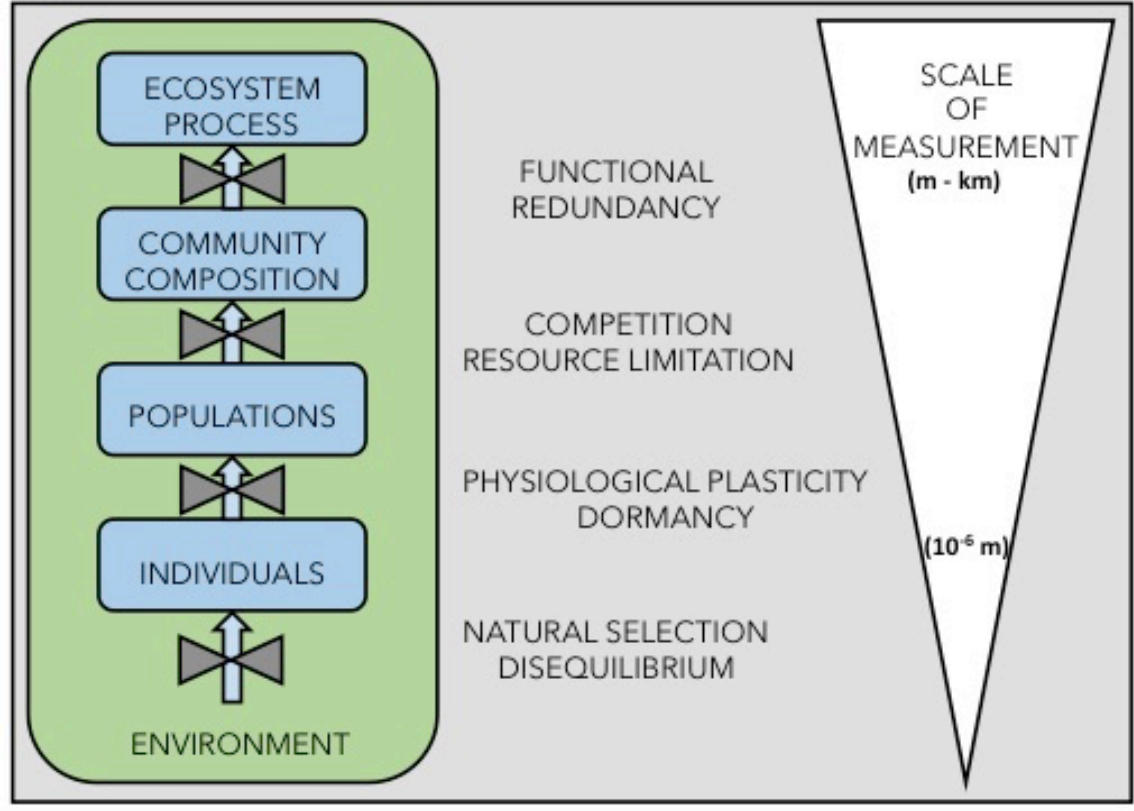

Figure 1 Diagram of microbial-ecosystem linkages A) how linkages are commonly conceptualized across levels of ecological organization and B) the series of ecological phenomena that create challenges when attempting to link metrics from one level of ecological organization to the other. 


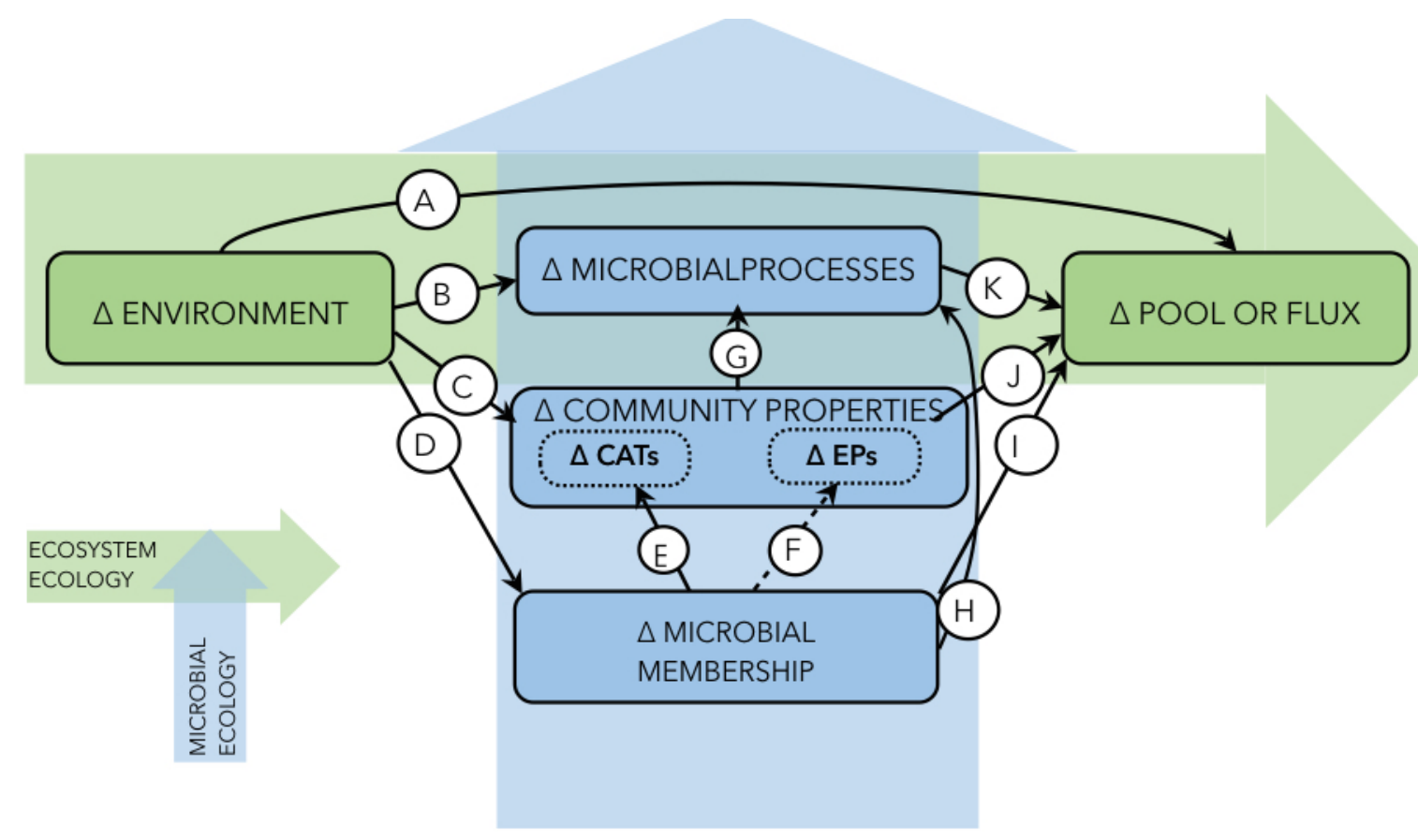

Figure 2 A conceptual map of the intersection between microbial (vertical) and ecosystem (horizontal) ecology illustrating each of the three categories of microbial characteristics (microbial processes, community properties, and microbial membership) as defined in the text. We argue for an increased focus on studies that elucidate pathways $\mathrm{E}, \mathrm{F}$, and $\mathrm{G}$. In addition, we note that pathways I and $\mathrm{J}$ are less likely to effectively incorporate microbiome characteristics into system-level science. The delta symbol in each category indicates an emphasis on how changes within a category may lead to a change in a connected category. The dotted arrow for letter $F$ denotes that many emergent properties cannot currently be linked to membership and is an important area for active research. CAT = community aggregated trait, $\mathrm{EP}=$ emergent property. 


\section{References}

1.Graham E. B., Wieder, W.R., Leff, J.R., Weintraub, S. W., Townsend, A.R., Cleveland, C.C., Philippot L., Nemergut D.R. (2014) Do we need to understand microbial communities to predict ecosystem function? A comparison of statistical models of nitrogen cycling processes Soil Biology and Biochemistry 68, 279-282

2.Graham E. B. et al. (2016) Microbes as engines of ecosystem function: when does community structure enhance predictions of ecosystem processes? Frontiers in Microbiology 7: 214 doi:10.3389/fmicb.2016.0021

3.Rocca J.D., Hall E.K., Lennon J.T., Evans S.E., Waldrop M.P., Cotner J.B., Nemergut D.R., Graham E.B., Wallenstein M.D. (2015) Relationships between protein-encoding gene abundance and corresponding process are commonly assumed yet rarely observed ISME J. 9: 1693-1699

4.Bier R.L., E.S. Bernhardt, CM Boot, EB Graham, EK Hall, JT Lennon, D Nemergut, B Osborne, C Ruiz-González, JP Schimel, MP Waldrop, MD. Wallenstein (2015) How are we forging conceptual, analytical, and mechanistic links between microbial community structure and ecosystem process? FEMS microbiology ecology FEMS microbiology ecology 91(10): fiv113

5. Falkowski, P.G., Fenchel, T., and DeLong, E.F. (2008) The microbial engines that drive earth's biogeochemical cycles Science 320: 1034-1038

6.Felip M., Pace M.L., Cole, J.J. (1996) Regulation of planktonic bacterial growth rates: The effects of temperature and resources. Microb. Ecol. 31(1): 15-28.

7. Lennon, J. T. and S. E. Jones (2011) Microbial seed banks: the ecological and evolutionary implications of dormancy. Nature Microbiology Reviews 9: 119-130.

8. Comte, J., Fauteux, L. and del Giorgio, P.A. (2013) Links between Metabolic Plasticity and Functional Redundancy in Freshwater Bacterioplankton Communities. Frontiers in Microbiology 4: 112. doi: 10.3389/fmicb.2013.00112

9. Adams, H. E., Crump, B.C. and Kling, G.W. (2014) Metacommunity Dynamics of Bacteria in an Arctic Lake: The Impact of Species Sorting and Mass Effects on Bacterial Production and Biogeography. Frontiers in Microbiology 5 (2014): 82. 10.3389/fmicb.2014.00082

10. Schimel, J.P., J. Bennett, and N. Fierer. (2005) Microbial community composition and soil N cycling: is there really a connection? In: Biological diversity and function in soils. Bardgett, R.D., D.W. Hopkins, and M.B. Usher (Eds.) Cambridge University Press. pp. 171-188

11. Schimel, J.P. (1995) Ecosystem consequences of microbial diversity and community structure in Arctic and Alpine Biodiversity: Patterns, Causes and Ecosystem Consequences Ecological Studies Volume 113, pp 239-254

12. Lenhart K., M. Bunge, S. Ratering, T. R. Neu, I. Schüttmann, M. Greule, C. Kammann, S. Schnell, C. Müller, H. Zorn \& F. Keppler. (2012) Evidence for methane production by saprotrophic fungi Nature Comm. 3:1046

13. Hagstroem, A., Azam, F., Berg, C., Zweifel U.L. (2017) Isolates as models to study bacterial ecophysiology and biogeochemistry Aquat. Microb. Ecol. 80:1527

14. Larkin, A.A, and Martiny, A. (2017) Microdiversity shapes the traits, niche space and biogeography of microbial taxa Environ. Microbiol. Rep. 9(2): 55-70 doi: 10.111/1758-2229 
15. German D.P., Marcelo, K.R.B., Stone, M.M and Allison, S.D. (2012) The Michaelis-Menten kinetics of soil extracellular enzymes in response to temperature: a cross-latitudinal study Global Change Biology 18: 1468-1479

16. Hall, E. K., A. R. Dzialowski, S. M. Stoxen, and J. B. Cotner. (2009) The effect of temperature on the coupling between phosphorus and growth in lacustrine bacterioplankton communities. Limnol. and Oceanogr. 54: 880-889.

17.Manzoni, S., R. B. Jackson, J. A. Trofymow, and A. Porporato. (2008) The global stoichiometry of litter nitrogen mineralization. Science 321: 684-686.

18. Elser, J.J., Chrzanowski, T.H., Sterner, R.W., Schampel, J.H., Foster D.K. (1995) Elemental ratios and the uptake and release of nutrients by phytoplankton and bacteria in three lakes of the canadian shield Microbial Ecology 29: 145-162

19. Goldman, J.C., Caron, D.A., and Dennett, M.R. (1987) Regulation of gross growth efficiency and ammonium regeneration in bacteria by substrate $\mathrm{C}: \mathrm{N}$ ratio Limnology and Oceanography 32 (6): 1239-1252

20.Jensen, B.D., Wise, K.E. and Odegard G.M. 2015 Simulation of the Elastic and Ultimate Tensile Properties of Diamond, Graphene, Carbon Nanotubes, and Amorphous Carbon Using a Revised ReaxFF Parametrization J. Phys. Chem. A, 119 (37): 9710-9721

21.Salt, G.W. (1979) A comment on the use of the term emergent properties American Naturalist 113: 145-148

22. Konopka, A. (2009) What is microbial community ecology? ISME J., 3 (11): 1223-12230

23. Battin, T., L. A. Kaplan, L. Newbold, X. Cheng, and C. Hansen. (2003) Effects of current velocity on the nascent architecture of stream microbial biofilms. Appl. and Env. Microbiol. 69: 5443-5452.

24. Martiny JBH, Jones SE, Lennon JT, Martiny AC (2015) Microbiomes in light of traits: a phylogenetic perspective. Science 350: doi: 10.1126/science.aac9323

25. Ruiz-González, C., Niño-García, J. P., Lapierre, J. F., \& del Giorgio, P. A. (2015) The quality of organic matter shapes the functional biogeography of bacterioplankton across boreal freshwater ecosystems. Global Ecology and Biogeography 24(12): 1487-1498.

26. Fierer, N., A. Barberán, Laughlin, D. (2014) Seeing the forest for the genes: Using metagenomics to infer the aggregated traits of microbial communities. Frontiers in Microbiology 5: 614

27. Judd, C.R., Koyama, A., Simmons, M.P., Brewer, P., and von Fischer, J.C. (2016) Co-variation in methanotroph community composition and activity in three temperate grassland soils Soil Biol. and Biochem. 95:78-86

28. Grilli, J., Barabas G., Michalska-Smith, M.J., and Allesina, S. (2017) Higher-order interactions stabilize dynamics in competitive network models Nature. 2017 548: 210-213

29. Newton, R. J, S. E. Jones, A. Eiler, K. D McMahon, Bertilsson, S. (2011) A guide to the natural history of freshwater lake bacteria. Microbiol. and Molec. Biol. Rev. 75:1, doi:10.1128/MMBR.00028

30. Hug, L.A., Baker, B.J., Anantharaman, K., Brown, C.T., Probst, A.J., Castelle, C.J., Butterfield, C.N., Hernsdorf, A.W., Amano, Y., Ise, K., Suzuki, Y., Dudek, N., Relman, D.A., Finstad, K.M., Amundson, R., Thomas, B.C., Banfield, J.F. (2016) 1: 16048 doi: 10.1038/nmicrobiol.2016.48 
31. Martiny, J.B.H., B. Bohannan, J. Brown, R. Colwell, J. Fuhrman, J. Green, M.C. Horner-Devine, M. Kane, J. Krumins, C. Kuske, P. Morin, S. Naeem, L. Ovreas, A.-L. Reysenbach, V. Smith, J. Staley. (2006) Microbial biogeography: Putting microorganisms on the map. Nature Reviews Microbiology 4: 102-112

32. Louca, S., Polz, M.F., Mazel, F., Albright, M.B.N., Huber, J. A., O’Connor, M.I., Ackermann, M. Hahn, A.S., Srivastava, D.S., Crowe, S.A., Doebeli, M. and Parfrey, L. W. 2018 Function and functional redundancy in microbial systems https://doi.org/10.1038/s41559-018-0519-1

33. Lennon JT, Aanderud ZA, Lehmkuhl BK, Schoolmaster DR (2012) Mapping the niche space of soil microorganisms using taxonomy and traits. Ecology 93: 1867-1879

34. Treseder KK, Lennon JT (2015) Fungal traits that drive ecosystem dynamics. Microbiology and Molecular Biology Reviews 79: 243-262

35. Czechowska, K., Johnson, D.R., van der Meer, J.R. (2008) Use of flow cytometric methods for single-cell analysis in environmental microbiology Curr. Opin. In Microbiol. 11:3, 205-212

36. Galand P.E., L. Alonso-Sáez, S. Bertilsson, C. Lovejoy, and E. O. Casamayor (2013) Contrasting activity patterns determined by BrdU incorporation in bacterial ribotypes from the Arctic Ocean in winter Front Microbiol. (2013); 4: 118

37. Biller, S.J., Berube, P.M., Lindell, D., Chisholm, S.W. (2015) Procholorcoccus: The structure and function of collective diversity Nature Reviews Microbiology 13:13-27

38. Brewer, T.E., Handley, K.M., Carini, P., Gilbert, J.A., Fierer, N. (2016) Genome reduction in an abundant and ubiquitous soil bacterium 'Candidatus Udaeobacter copiosus' Nature Microbiology 2: 16198

39. Neufeld J.D., Vohra J., Dumont M.G., Lueders T., Manefield M., Friedrich M.W., Murrell J.C. (2007) DNA stable-isotope probing. Nat Protoc. 2:4, 860-866

40. Wagner, M. (2009) Single cell ecophysiology of microbes as revealed by Raman microspectroscopy or secondary ion mass spectrometry imaging. Annu Rev Microbiol 63:411-429.

41. Pester, M., Bittner, N., Deevong, P., Wagner, M., and Loy, A. (2010) A 'rare biosphere' microorganism drives sulfate reduction in a peatland. ISME J. 4:1591-1602.

42. Behrens, S., Kappler, A., Obst M. (2012) Linking environmental processes to the in situ functioning of microorganisms by high-resolution secondary ion mass spectrometry (NanoSIMS) and scanning transmission X-ray microscopy (STXM) Env. Microbiol. 14(11): 2851-69

43. Norland S, Fagerbakke K, Heldel M. (1995) Light element analysis of individual bacteria by X-ray microanalysis. Appl. Environ. Microbiol. 61:1357-62.

44. Wilbanks, E.G., Jaekel, U., Salman, V., Humphrey, P.T., Eisen, J.A., Faciotti, M.T., Buckley, D.H., Zinder, S.H., Druschel, G.K., Fike, D.A., Orphan,V.J., (2014) Microscale sulfur cyclingin the photophrophic pink berry consortia of the Sippewissett Salt Marsh Environ. Micro 16: 3398-3415

45. Hall, E.K., Maixner F., Franklin O., Daims, H., Richter, A. and Battin, T. (2011) Linking microbial and ecosystem ecology using ecological stoichiometry: A synthesis of conceptual and empirical approaches Ecosystems 14: 261-273 